International Journal of Pure and Applied Mathematics

Volume $100 \quad$ No. 1 2015, 75-80

ISSN: 1311-8080 (printed version); ISSN: 1314-3395 (on-line version)

url: http://www.ijpam.eu

doi: http://dx.doi.org/10.12732/ijpam.v100i1.7

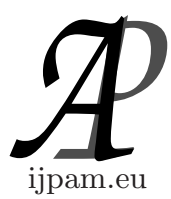

\title{
ON OPTIMAL DERIVATIVE ERROR BOUNDS FOR LAGRANGE INTERPOLATION
}

\author{
F. Dubeau \\ Mathematics Department \\ University of Sherbrooke \\ 2500 University Boul., Sherbrooke (Qc), CANADA, J1K 2R1
}

\begin{abstract}
We analyze the approximation of the derivative of a function by considering the derivative of its Lagrange interpolation polynomial. Optimal truncation errors bounds are established by a direct approach using Peano's kernels and depend on the regularity of the function.
\end{abstract}

AMS Subject Classification: 65D25, 65D05, 26A46

Key Words: numerical differentiation, Lagrange interpolation, Taylor's expansion, Peano's kernel

\section{Introduction}

Let $I=[a, b]$ and $C^{l}(I)$ be the set of continuously differentiable functions up to order $l$ on $I$. Let $p \in[1,+\infty]$, and let the set of absolutely continuous function on $I$ be defined by

$$
A C^{l+1, p}(I)=\left\{\begin{array}{l|l}
f \in C^{l}(I) & \begin{array}{l}
(a) f^{(l+1)} \in L^{p}(I), \text { and } \\
(b) f^{(l)}(s)=f^{(l)}(r) \\
\\
+\int_{r}^{s} f^{(l+1)}(y) d y, \forall r, s \in I
\end{array}
\end{array}\right\} .
$$

Let us assume that a set of nodes $\left\{x_{i}\right\}_{i=0}^{n}$ such that $a \leq x_{0}<\cdots<x_{i}<$ $\cdots<x_{n} \leq b$ is given. For a continuous function $f(\cdot)$ on $I$, let $L(x)$ be its

Received: December 3, 2014

(c) 2015 Academic Publications, Ltd. url: www.acadpubl.eu 
Lagrange interpolation polynomial satisfying

$$
L\left(x_{i}\right)=f\left(x_{i}\right) \text { for } i=0, \ldots, n .
$$

Using the Lagrange interpolation formula, we have

$$
f(x) \approx L(x)=\sum_{i=0}^{n} f\left(x_{i}\right) l_{n, i}(x)
$$

where $l_{n, i}(x)$ is the $i$ th Lagrange interpolating polynomial of degree $n$ given by

$$
l_{n, i}(x)=\prod_{\substack{j=0 \\ j \neq i}}^{n} \frac{\left(x-x_{j}\right)}{\left(x_{i}-x_{j}\right)} \quad \text { for } \quad i=0, \ldots, n
$$

To approximate the $k$ th derivative, we use

$$
f^{(k)}(x) \approx L^{(k)}(x)=\sum_{i=0}^{n} f\left(x_{i}\right) l_{n, i}^{(k)}(x)
$$

for $k=1, \ldots, n$. The troncature error for the $k$ th derivative process is defined by

$$
R_{n, l}^{(k)}(f ; x)=f^{(k)}(x)-L^{(k)}(x)=f^{(k)}(x)-\sum_{i=0}^{n} f\left(x_{i}\right) l_{n, i}^{(k)}(x) .
$$

Since (1) and (2) are based on the Lagrange interpolation polynomial, they are exact for polynomials of degree $\leq n$.

In this paper we would like to present optimal error bounds for $R_{n, l}^{(k)}(f ; x)$ which depends on the regularity of $f(\cdot)$. Our results extend results presented in [4], [5], [3], and [6] for regular functions $f(\cdot) \in A C^{n+1, \infty}(I)$.

\section{Taylor's Expansion}

The Taylor's expansion of $f \in A C^{l+1, p}(I)$ of order $l+1$ around $c \in I$, see [2] and [7], is

$$
f(x)=\sum_{j=0}^{l} \frac{f^{(j)}(c)}{j !}(x-c)^{j}+\int_{a}^{b} f^{(l+1)}(y) K_{T, l}(y ; x, c) d y
$$


where $K_{T, l}(y ; x, c)$ is the kernel given by

$$
K_{T, l}(y ; x, c)=\frac{1}{l !}\left[(x-y)_{+}^{l} \mathbf{1}_{[c, b]}(y)+(-1)^{l+1}(y-x)_{+}^{l} \mathbf{1}_{[a, c]}(y)\right],
$$

This kernel is a piecewise polynomial function of degree $l$, and consequently $K_{T, l}(\cdot ; x, c) \in L^{\infty}(I)$. In this expression, if $E$ is a set, then

$$
\mathbf{1}_{E}(y)=\left\{\begin{array}{lll}
1 & \text { if } & y \in E, \\
0 & \text { if } & y \notin E .
\end{array}\right.
$$

\section{Truncation Error}

For any integer $l$ such that $k \leq l \leq n$, let $f(x) \in A C^{l+1, p}(I)$. Since the process (2) is exact for polynomials of degree $\leq n$, using a Taylor's expansion (3) of order $l+1$ for $f(\cdot)$, the truncation error becomes

$$
R_{n, l}^{(k)}(f ; x)=\int_{a}^{b} f^{(l+1)}(y) K_{n, l}^{(k)}(y ; x, c) d y
$$

where $K_{n, l}^{(k)}(y ; x, c)$ is the Peano kernel associated to the process, given by

$$
\begin{aligned}
K_{n, l}^{(k)}(y ; x, c) & =R_{n, l}^{(k)}\left(K_{T, l}(y ; \cdot, c) ; x\right) \\
& =K_{T, l-k}(y ; x, c)-\sum_{i=0}^{n} l_{n, i}^{(k)}(x) K_{T, l}\left(y ; x_{i}, c\right) .
\end{aligned}
$$

Let $q \in[1,+\infty]$ be the conjugate of $p$ such that $\frac{1}{p}+\frac{1}{q}=1$. Using Holder's inequality, we get from (4)

$$
\left|R_{n, l}^{(k)}(f ; x)\right| \leq\left\|f^{(l+1)}(\cdot)\right\|_{p, I}\left\|K_{n, l}^{(k)}(\cdot ; x, c)\right\|_{q, I} .
$$

Hence we have proved our main result which point out the dependance of the error in terms of the regularity of the function.

Theorem 1. Since $R_{n, l}^{(k)}(f ; x)=0$ for any polynomial of degree $\leq n$, then (5) holds for any $f \in A C^{l+1, p}(I)$ for $k \leq l \leq n$.

Theorem 2. For each $x \in I$, the bound given by (5) is the best one. 
Proof. To show we can have equality in (4) and (5), we use a standard construction for $f^{(l+1)}(\cdot)$ given in [1]. Indeed, for $1<p \leq \infty(1 \leq q<\infty)$, let $f(\cdot) \in A C^{l+1, p}(I)$ be such that

$$
f^{(l+1)}(y)= \begin{cases}\left|K_{n, l}^{(k)}(y ; x, c)\right|^{q-2} K_{n, l}^{(k)}(y ; x, c) & \text { for } \quad K_{n, l}^{(k)}(y ; x, c) \neq 0, \\ 0 & \text { for } \quad K_{n, l}^{(k)}(y ; x, c)=0 .\end{cases}
$$

Then we get an equality

$$
\int_{a}^{b} f^{(l+1)}(y) K_{n, l}^{(k)}(y ; x, c) d y=\left\|f^{(l+1)}(\cdot)\right\|_{p, I}\left\|K_{n, l}^{(k)}(\cdot ; x, c)\right\|_{q, I}
$$

because

$$
\left\|f^{(l+1)}(\cdot)\right\|_{p, I}=\left\|K_{n, l}^{(k)}(\cdot ; x, c)\right\|_{q, I}^{q-1}
$$

and

$$
\int_{a}^{b} f^{(l+1)}(y) K_{n, l}^{(k)}(y ; x, c) d y=\left\|K_{n, l}^{(k)}(\cdot ; x, c)\right\|_{q, I}^{q} .
$$

For $p=1(q=\infty)$, we suppose that $\left\|K_{n, l}^{(k)}(\cdot ; x, c)\right\|_{\infty, I}>0$. Let us consider $0<\epsilon<\left\|K_{n, l}^{(k)}(\cdot ; x, c)\right\|_{\infty, I}$, and let us define the set $A_{\epsilon}$ by

$$
A_{\epsilon}=\left\{y \in I|| K_{n, l}^{(k)}(y ; x, c) \mid \geq\left\|K_{n, l}^{(k)}(\cdot ; x, c)\right\|_{\infty, I}-\epsilon\right\} .
$$

This set is such that its Lebesque's measure is $0<\mu\left(A_{\epsilon}\right) \leq b-a$. If we set

$$
f^{(l+1)}(y)= \begin{cases}\left|K_{n, l}^{(k)}(y ; x, c)\right|^{-1} K_{n, l}^{(k)}(y ; x, c) & \text { for } y \in A_{\epsilon}, \\ 0 & \text { elsewhere }\end{cases}
$$

then

$$
\begin{aligned}
\left\|f^{(l+1)}(\cdot)\right\|_{1, I}\left(\left\|K_{n, l}^{(k)}(\cdot ; x, c)\right\|_{\infty, I}-\epsilon\right) & \leq \int_{a}^{b} f^{(l+1)}(y) K_{n, l}^{(k)}(y ; x, c) d y \\
& \leq\left\|f^{(l+1)}(\cdot)\right\|_{1, I}\left\|K_{n, l}^{(k)}(\cdot ; x, c)\right\|_{\infty, I},
\end{aligned}
$$

because

$$
\left\|f^{(l+1)}(\cdot)\right\|_{1, I}=\mu\left(A_{\epsilon}\right) .
$$

Since $\epsilon>0$ can be arbitrary small, the result follows. 
Theorem 3. The kernel $K_{n, l}^{(k)}(y ; x, c)$ does not depend on $c$

Proof. For $c$ and $\tilde{c}$ we have

$$
\int_{a}^{b} f^{(l+1)}(y)\left[K_{n, l}^{(k)}(y ; x, c)-K_{n, l}^{(k)}(y ; x, \tilde{c})\right] d y=0,
$$

for any $f^{(l+1)}(\cdot) \in L^{p}(I)$ and $p \in[1, \infty]$. It follows that for each given fixed $x$

$$
K_{n, l}^{(k)}(y ; x, c)=K_{n, l}^{(k)}(y ; x, \tilde{c})
$$

almost everywhere with respect to $y$. Moreover, the two expression can be different only at a finite number of $y$ values because they are two piecewise polynomial functions.

Finally, since $\left\|K_{n, l}^{(k)}(\cdot ; x, c)\right\|_{q, I}$ is a continuous function with respect to $x \in I$, its maximum value exists, is finite, and there exists at least one $x^{*} \in I$ such that

$$
\max _{x \in I}\left\|K_{n, l}^{(k)}(\cdot ; x, c)\right\|_{q, I}=\left\|K_{n, l}^{(k)}\left(\cdot ; x^{*}, c\right)\right\|_{q, I} .
$$

\section{Acknowledgments}

This work has been financially supported by an individual discovery grant from NSERC (Natural Sciences and Engineering Research Council of Canada).

\section{References}

[1] R.A. Adams, Sobolev Spaces, Academic Press, New York (1975).

[2] E. Asplund and L. Bungart, A first course in integration, Holt, Rinehart and Winston, New York (1966).

[3] M.S. Floater, Error formulas for divided difference expansions and numerical differentiation, Journal of Approximation Theory, 122 (2003), 1-9, doi: 10.1016/S0021-9045(03)00025-X.

[4] G.W. Howell, Derivative error bounds for Lagrange interpolation: An extension of Cauchy's bound for the error of Lagrange interpolation, Journal of Approximation Theory, 67 (1991), 164-173, doi: 10.1016/00219045(91)90015-3. 
[5] A. Shadrin, Error bounds for Lagrange interpolation, Journal of Approximation Theory, 80 (1995), 25-49, doi: 10.1006/jath.1995.1003.

[6] A. Spitzbart and N. Macon, Numerical differentiation formulas, The American Mathematical Monthly, 64 (1957), 721-723, doi: 10.2307/2309751.

[7] L.L. Schumaker, Spline functions basic theory, Wiley, New York (1981). 